Submitted to Journal of Synchrotron radiation

as a Full Article

\title{
SUITE OF THREE PROTEIN CRYSTALLOGRAPHY BEAMLINES WITH SINGLE SUPERCONDUCTING BEND MAGNET AS THE SOURCE
}

\author{
DISCLAIMER
}

This document was prepared as an account of work sponsored by the United States Government. While this document is believed to contain correct information, neither the United States Government nor any agency thereof, nor The Regents of the University of California, nor any of their employees, makes any warranty, express or implied, or assumes any legal responsibility for the accuracy, completeness, or usefulness of any information, apparatus, product, or process disclosed, or represents that its use would not infringe privately owned rights. Reference herein to any specific commercial product, process, or service by its trade name, trademark, manufacturer, or otherwise, does not necessarily constitute or imply its endorsement, recommendation, or favoring by the United States Government or any agency thereof, or The Regents of the University of California. The views and opinions of authors expressed herein do not necessarily state or reflect those of the United States Government or any agency thereof or The Regents of the University of California. 


\title{
SUITE OF THREE PROTEIN CRYSTALLOGRAPHY BEAMLINES WITH SINGLE SUPERCONDUCTING BEND MAGNET AS THE SOURCE
}

\author{
Alastair A. MacDowell, ${ }^{a^{*}}$ Rich S. Celestre, ${ }^{a}$ Malcolm Howells, ${ }^{a}$ Wayne McKinney, ${ }^{a}$ James \\ Krupnick,, Daniella Cambie, ${ }^{b}$ Edward E. Domning, ${ }^{b}$ Robert M. Duarte, ${ }^{b}$ Nicholas Kelez, ${ }^{b}$ David \\ W. Plate, ${ }^{b}$ Carl W. Cork, ${ }^{c}$ Thomas N. Earnest, ${ }^{c}$ Jeffery Dickert, ${ }^{c}$ George Meigs, ${ }^{c}$ Corie Ralston, ${ }^{c}$ \\ James M. Holton, ${ }^{\text {c,d }}$ Tom Alber, ${ }^{d}$ James M. Berger, ${ }^{d}$ David A. Agard, ${ }^{e}$-Howard A. Padmore. ${ }^{a}$ \\ ${ }^{a}$ Advanced Light Source, Lawrence Berkeley National Lab., 1 Cyclotron Road, Berkeley, CA 94720, USA \\ ${ }^{b}$ Engineering Div., Lawrence Berkeley National Lab., 1 Cyclotron Road, Berkeley, CA 94720, USA \\ ${ }^{c}$ Physical Biosciences, Lawrence Berkeley National Lab., 1 Cyclotron Road, Berkeley, CA 94720, USA \\ ${ }^{d}$ Dept. of Molecular and Cell Biology, 229 Stanley Hall, University of Berkeley, Berkeley, California, 94720, USA \\ ${ }^{e}$ Biochemistry/Biophysics and Pharmaceutical Chemistry, University of San Francisco, San Francisco, California, \\ 94143, USA \\ *Email:aamacdowell@lbl.gov
}

\section{Synopsis}

Three near identical protein crystallography beamlines with a single 6 Tesla peak field superconducting dipole bend magnet as the source have been built at the $1.9 \mathrm{GeV}$ Advanced Light Source. The design and performance of this new facility is described.

\section{Abstract}

At the Advanced Light Source (ALS), three protein crystallography (PX) beamlines have been built that use as a source one of the three 6 Tesla single pole superconducting bending magnets (superbends) that were recently installed in the ring. The use of such single pole superconducting bend magnets enables the development of a hard x-ray program on a relatively low energy $1.9 \mathrm{GeV}$ ring without taking up insertion device straight sections. The source is of relatively low power, but due to the small electron beam emittance, it has high brightness. X-ray optics are required to preserve the brightness and to match the illumination requirements for protein crystallography. This was achieved by means of a collimating premirror bent to a plane parabola, a double crystal monochromator followed by a toroidal mirror that focuses in the horizontal direction with a 2:1 demagnification. This optical arrangement partially balances aberrations from the collimating and toroidal mirrors such that a tight focused spot size is achieved. The optical properties of the beamline are an excellent match to those required by the small protein crystals that are typically measured. The design and performance of these new beamlines are described.

Keywords: x-ray beamline, protein crystallography, superbend, invar mirror, double crystal monochromator. 


\section{Introduction}

The Advanced Light Source is a relatively low-energy, $3^{\text {rd }}$ generation synchrotron optimized for the production of VUV and soft x-ray light from undulators. However, it was realized early on in the development of the ALS that a multipole wiggler source with good optics would provide excellent performance for protein crystallography at up to $15 \mathrm{KeV}$. Based on the needs of the local community, a wiggler beamline with 1 MAD (multiwavelengthanomolous-diffraction) central line and 2 fixed wavelength side stations was constructed, with initial commissioning of the central station late in 1997 (Earnest et al., 1996). The success of this beamline 5.0.2 MAD station generated calls from the community for additional beamlines. Because additional straight sections for wigglers were not available, an alternative solution was required. One of the solutions considered was based on an research and development project at Lawrence Berkeley National Laboratory to produce superconducting replacements for some of the bend magnets of the ALS (Robin et al., 2001)

This early work showed that a magnet with a peak field of 6 Tesla could be built and operated reliably in a storage ring environment. The design allowed for 4 beamlines per magnet, with inboard and outboard pairs of tangent points at field strengths of 5.29 and 4.37 Tesla. These fields increased the critical energy from $3 \mathrm{KeV}$ for a 1.25 Tesla normal conducting magnet to 11.5 and $12.7 \mathrm{KeV}$, respectively. Introducing high field bend magnets into the ALS lattice increased the horizontal emittance. To compensate for this effect, the number of high field magnets was limited to three. This design resulted in a doubling of the horizontal emittance from $6 \times 10^{-9}$ to $1.2 \times 10^{-8}$ meter.radians, but running with a small dispersion in the straights was shown to reduce this to close to the original value $\left(6.8 \times 10^{-9}\right.$ meter radians). Because installation of the high field magnets reduced the symmetry of the machine from 12 to 3, significant additional work had to be done to ensure stable dynamics of the machine with good lifetime and injection efficiency. (Steier et al., 2001)

A further milestone in establishing the viability of this source for protein crystallography was the validation of the radiation damage model of Henderson (Henderson, 1990) by Glaeser et al. (Glaeser et al., 2000). This work showed that for 'typical' crystals with $10 \mathrm{~nm}$ unit cell size, at a wavelength of $0.1 \mathrm{~nm}$, an integrated incident flux of $10^{10}$ photons/micron ${ }^{2}$ caused sufficient radiation damage to reduce the intensities of diffraction spots at $0.25 \mathrm{~nm}$ resolution by $50 \%$. Typical sizes of protein crystals are 100 micron and the flux from a superbend source with optimized beamline through a 100 micron pinhole is calculated at $\sim 4 \times 10^{11}$ photon/sec with a horizontal convergence onto the sample of 3 mrads. 
These parameters indicate that the photon dose limit will be reached in $\sim 200$ seconds. Considering the number of frames that could be recorded in this time, the readout time of state of the art detector systems, and the time required to mount and align crystals, this performance was considered sufficient for the majority of systems that the community wanted to study. However, it needs to be pointed out that the idea of a 'typical' crystal is not particularly valid. Radiation damage in cryofrozen proteins illuminated with x-rays is actually quite variable. Although the Henderson limit reflects an average, different types of crystals differ in radiation damage rates by at least a factor of 10 either way (Garman and Nave, 2002).

For crystals with very large unit cells where extreme collimation is required, the characteristics of undulators at high energy storage rings offer the best performance. Even in these challenging cases, however, the measured performance of the superbend beamlines at the ALS is significantly higher than the original ALS 5.0.2 wiggler beamline that has been extensively and successfully used for the study of several large-unit-cell problems including the ribosome structure (Yusupov et al., 2001).

Based on the expected characteristics of the superbends and the calculated performance of the optical systems, a suite of three near identical beamlines have been built over the last 3 years in sector 8 of the ALS. The design and performance of the beamlines and end station systems is reported here.

\section{The Superconducting single dipole bend magnet source}

The ALS storage ring lattice is 12 -fold symmetric with the basic magnet lattice of the triple bend achromat type (1-2 GeV Synchrotron Radiation Source - Conceptual Design Report, 1986). Three of the thirty six 1.27 Tesla warm, 10 degree, bending magnets (critical energy = $3 \mathrm{KeV}$ ) were replaced by 6 Tesla, superconducting, 10 degree, bending magnets (critical energy $12 \mathrm{KeV}$ ) (Robin et al., 2002, Tamura \& Robinson, 2002). Three symmetrically located sectors of the 12 sectors of the ALS were modified to accommodate the high field magnets. The schematic of the modification is shown in Fig.1. The central dipole, B2, in the sector was replaced by a superbend. The warm dipole magnets have a radial magnetic gradient and thus have a quadrupole focussing component. The superbend is a plain dipole, and two new quadrupoles were added on either side of the superbend to replace the vertical focusing provided previously by the gradient field. The superbend magnets themselves are of a warm bore construction, and they fit over slots machined into the existing sector arc chamber. A closed-cycle Sumitomo cryocooler was used to cool each magnet to liquid-helium 
temperature, eliminating the need for additional cryogens. In the event of a cryocooler failure, the system can be run from an external liquid helium supply. The vertically integrated synchrotron power output from the superbend source is 58 watts/horizontal $\mathrm{mrad} / 400 \mathrm{~mA}$ at $1.9 \mathrm{GeV}$. The electron source size is approximately $230 \times 30 ? \mathrm{~m}$ full width at half maximum (FWHM).

Retrofitting the storage ring with superconducting dipoles was the first ever operation of this type carried out on an operating synchrotron radiation source. The superbends are an essential part of the ring lattice and problems associated with them affect all users of the ring. The installation process involved two, 6-week shutdowns. All major components of the project except the superbend magnets (including the additional quadrupole magnets, cryogenics and control systems) were installed in the first shutdown. The superbend magnets were installed during the second shutdown. Extensive beam-dynamics studies were carried out beforehand, and all systems were modelled and tested prior to installation. The commissioning proved to be very successful. The first beam was injected within 5 minutes of the first attempt, and $100 \mathrm{~mA}$ was achieved within the first hour. After more than a year of use, the superbends appear transparent to the rest of the users on the ring.

\section{Beamline design}

The beamlines were designed to have the highest flux possible through a $100 ? \mathrm{~m}$ diameter pinhole with a $3 \mathrm{mrad}$ convergence angle in the 6-16 $\mathrm{KeV}$ energy range, consistent with reasonable cost and robust performance. Stable performance and rapid beam optimization were very important design considerations, given the expected high volume of use and rapid turnover of user groups. The $100 ? \mathrm{~m}$ pinhole represents the typical size of protein crystals produced nowadays. The beamlines were also optimized for MAD crystallography that requires precise and rapid switching of x-ray energy with reproducible beam intensity through the pinhole. The beamline design is shown schematically in Fig.2. It consists of the source, a vertically deflecting plane parabolic collimating mirror (grazing angle $=4.5 \mathrm{mrad}$, acceptance $=1.5 \times 0.5 \mathrm{mrad}$ (horizontal $(\mathrm{h}) \times$ vertical $(\mathrm{v}))$ that provides parallel radiation (in the vertical) for a double crystal monochromator (two flat crystals), followed by a toroidal focusing mirror, the sample and backstop. For beamline 8.2.2 the various distances of the beamline components from the source were $6.5,16.5,19.53$ and $29.3 \mathrm{~m}$ respectively for the plane parabola, monochromator, toroid and sample respectively. The two other beamlines use slightly different distances for spatial reasons, but are essentially identical. Such optical 
arrangements have been used extensively on second-generation sources where the larger source size reduces concerns about the large aberrations of the image due to the astigmatic nature of the source (infinity in the vertical and real in the horizontal - see Appendix).

We found by raytracing of perfect optics (optical slope errors = zero) using Shadow (Lai \& Cerrina, 1986) that when the toroidal mirror is used with parallel illumination in the vertical direction and a 2:1 demagnification in the horizontal, the main aberrations cancel and a high quality image results. This is shown in Fig.3, which shows the ray traced focus for different demagnifications of the M2 toroid. Even though the horizontal and vertical demagnification reduces from left to right across the figure, the fidelity of the focus is optimum at a demagnification close to 2.0. Current work (Howells et al., 2003) (see Appendix) indicates that this is due to the complete elimination of astigmatic coma for the 2:1 horizontal demagnification case. Using an electron source size of $230 \times 37$ ? m FWHM (h x $\mathrm{v}$ ), the focused spot size is ray traced to be $150 \times 63$ ? m FWHM (h x v) with a beamline acceptance of $1.5 \times 0.5 \mathrm{mrad}(\mathrm{h} \times \mathrm{v})$. Because diffraction-limited imaging with horizontal and vertical demagnifications of 2.0 and 0.62 , respectively, should produce an image of size $115 \mathrm{x}$ 48 ?m FWHM (h x v), this optical layout results in some brightness loss. With the sample phase space under-filled in the vertical, this flux loss is not significant. Moreover, the increase in exposure time needed to offset the reduction in flux is more than offset by the operation simplicity associated with the mechanical robustness of the design.

Other optical designs were considered. In particular, the leading alternative design comprised a standard sagittally focusing crystal arrangement with a vertically deflecting plane parabolic collimating mirror (as above) followed by a double crystal monochromator (first crystal flat and second crystal sagittally focusing) and a plane parabolic focusing mirror. This design yielded an excellent theoretical optical focus. With 1:1 horizontal and 2:1 vertical focusing, a spot profile at the sample of $239 \times 12$ ?m FWHM was ray traced. This is essentially just the geometrical source size, indicating that aberrations are very low in this design. The theoretical brightness at the image was about a factor of 2 better than the mirrorfocused solution we adopted. Considerable discussion was put in to which of the two designs to implement. The very thin and dynamic nature of the sagittally focusing crystal was considered a major drawback, because this design would require significant operational care to ensure peak effectiveness all the time, especially during MAD scans requiring frequent energy changes. Instead of choosing this theoretically perfect design, we adopted the mirrorfocused solution due to its greater simplicity and robustness. It should be noted that the focusing of the mirror-focused solution can be improved to give a more theoretically perfect 
image, but at the expense of a non-toroidal M2 mirror. For instance by making the M2 mirror a bent cone, near perfect imaging can be achieved. We believe that the increasing capabilities of optical manufacturers through precise measurement and programmed polishing will make this is a viable solution for a future upgrade.

\subsection{Optics}

To retain the brightness of the source, the optical slope errors need consideration. Tangential slope errors of all the optics will degrade the vertical image size. Sagittal slope errors have minimal effect on horizontal image size due to the grazing incident nature of the optics. Tangential slope errors of the M2 toroidal mirror will degrade the vertical image size by the product of the mirror-to-focus distance and twice the slope error. For the upstream optical elements, the effect of slope errors is to disperse the rays entering the toroidal focusing element. These photons will be focused in the vertical and differ from the correct focus by the product of the angular deviation and the M2 mirror-to-image distance. Therefore slope errors on all four optical elements can contribute equally to degrading the focus. Thermal distortions will affect the slope errors of the M1 mirror and the first crystal. Manufacturing defects will affect the slope errors of the M1 and M2 mirrors. The second crystal, if mounted strain free, can be considered perfect for the slope error analysis. The ray tracing of slope free optics indicated a vertical spot size of $150 \times 63$ ? m FWHM at the typical image distance from M2 of $10 \mathrm{~m}$ for the three beamlines. If we are prepared to tolerate an optical slope error equal to the calculated theoretical vertical spot size, then the tangential slope error of the optics can contribute 63 ? $\mathrm{m}$ FWHM, this leads to a vertical spot size some 1.41 times larger, which at 89 ? $\mathrm{m}$ is still within the specification of $100 ? \mathrm{~m}$. If we assume that the three elements noted above contribute equally in a quadratic manner, then the slope error image contribution for each element would be 36 ? m FWHM $\left(63 \times 3^{-1 / 2}\right)$. Taking into account angle doubling on reflection and the $10 \mathrm{~m}$ image distance from $\mathrm{M} 2$, the tangential slope error budget for each of the 3 elements is 1.8 ? rads FWHM (0.8 urads rms). This specification is quite challenging, but as the rest of this section will show, it is practical with current water-cooled optical technology

The M1 plane paraboloid mirror was made from a flat nickel plated invar mirror bent to the shape of a plane parabola by means of a mechanical bender. Adequate cooling was achieved using five, $6 \mathrm{~mm}$ holes, gun drilled down the length of the mirror with a skin thickness of $2.5 \mathrm{~mm}$. End caps were simply welded over the ends. The use of complicated 
water-to-vacuum joints was avoided by using two, upstream, 125-?m-thick, beryllium windows to separate the storage ring and beamline vacuums. The invar used $(36 \% \mathrm{Ni}+64 \%$ $\mathrm{Fe}$ ) has a very low coefficient of thermal expansion in the neighborhood of room temperature $\left(5 \times 10^{-7} /{ }^{\circ} \mathrm{C}\right)$. This stability allows it to perform favorably with an absorbed heat load of 76.2 watts, despite its rather poor thermal conductivity $\left(10.4 \mathrm{~W} / \mathrm{m} /{ }^{\circ} \mathrm{C}\right)$. Peak power density absorbed was 0.0018 watts $/ \mathrm{mm}^{2}$ on axis. A finite element analysis of the slope error deviation indicated that it would change 0.7 ? rad rms between no beam and full beam (400mA). Given that the ALS operates between 200 and $400 \mathrm{~mA}$, and the thermal bump is monotonic (i.e. the slope deviation can be corrected by re-bending M2) this thermal bump was considered satisfactory.

The grazing angle used was $4.5 \mathrm{mrad}$, with a $8 \mathrm{~nm}$ coating of Rhodium over $25 \mathrm{~nm}$ of Platinum. The reflectivity of this bilayer is shown in Fig.4. along with the reflectivity of single layers of Rhodium and Platinum at the same grazing angle. The reflectivity cut-off of this bilayer is extended $\sim 2.5 \mathrm{KeV}$ past that for a simple Rhodium mirror, because the harder radiation penetrates the Rhodium and reflects from the Platinum underlayer. The L edges of the Platinum are highly suppressed, because at this lower energy, the x-rays reflect principally from the upper Rhodium surface. This bilayer concept enables higher mirror performance at a given grazing angle with only a minor increase in coating cost.

The Invar mirrors were delivered from the manufacturer in two lots. The first lot, with slope errors of 1.3-1.6 ? rad rms and a finish of $1.3-1.7 \mathrm{~nm} \mathrm{rms}$, did not meet the required specification. To meet the construction schedule, however, they were pressed into service with hopes that the second batch would be more promising. The second batch, however, was shown by the ALS metrology group to have blisters on the surface due to Nickel delamination from the Invar substrate. This delamination was caused by improper cleaning and surface preparation. After a detailed review of how these mirrors should be fabricated (Howells et. al. (2002)), new optic vendors (InSync) and plating vendors (Acteron) were contracted to build a third batch of mirrors. Because this Invar mirror technology was new to synchrotron radiation optics, we adopted in parallel a more conservative engineering strategy. This fall-back strategy employed a conventional side cooled silicon mirror design. Cooling was achieved using water cooled copper bars held along the mirror sides with a 50-100 ? m gap filled with Gallium-Indium-Tin eutectic liquid metal. Finite element modeling indicated that this arrangement produced identical performance to the internally cooled invar mirrors with regard to the thermal bump, if the assumption was made that the thermal resistance of the mirror to cooling plate liquid metal interface was negligible. Metrology measurements indicated that 
both the new batch of invar mirrors and the silicon mirrors met specifications. Beamline 8.3.1 had an Invar M1 mirror installed with a slope error of 0.8 ? rad rms and a finish of $0.9 \mathrm{~nm} \mathrm{rms}$. Beamlines 8.2.1 and 8.2.2 had silicon M1 mirrors installed with slightly better slope errors of 0.65 ? rad rms and a finish of $0.5 \mathrm{~nm} \mathrm{rms}$.

The next optical elements are the two flat $\mathrm{Si}(111)$ crystals in a Kohzu APM type double-crystal monochromator. The first crystal receives a total power load of $\sim 45$ watts with a maximum power density of $0.25 \mathrm{watts} / \mathrm{mm}^{2}$ at $12.4 \mathrm{KeV}$. At these power levels, internal water-cooling is adequate, but care has to be taken with the design of the water channels. For example, it was found that with a standard longitudinal configuration of cooling channels, the thermal distortion was out of specification. Figure 5 shows sections of the crystal. Five 1.5 $\mathrm{mm}$ diameter holes were drilled through the crystal transverse to the beam direction. The minimum hot wall thickness is $1.5 \mathrm{~mm}$ for the central hole and increases for the outer holes. In this way, the gaussian heat load on the crystal was dissipated more aggressively in the center where the power density is highest and less so in the wings. The separation and depth of the through holes was selected to minimize the thermal bump at $12.4 \mathrm{KeV}$. A finite element analysis of the slope error at $12.4 \mathrm{KeV}$ indicated that for the beam on/off situation, the slope change is 0.7 ? rad rms. This value was within specification of the slope error. Due to the very small angular size of the source, the slope errors required from the thermally distorted crystal are much less than that of the single crystal rocking curve width (30 ? rad FWHM at 12.4 $\mathrm{KeV})$. In general, preserving brightness on third-generation sources requires slope errors to be much less than rocking curve widths. The diffracting face of the crystal was recessed between two, $7 \mathrm{~mm}$ thick, silicon wings that run the length of the crystal. Invar water plenums were glued to both sides of the crystal with water circulated through the five small holes and returned through the large lower hole. The silicon wings provided a contact area for the glue. When using glue, the concern is failure due to radiation exposure. The glue used was a thermoconductive epoxy resin of type E707 from Épotecny. This adhesive has been used successfully at the ESRF (Freund, 2000) for 1-2 years. The glue radiation exposure due to Compton scattering was calculated (Howells, 1999) and the estimated glue lifetime was found to be $>100$ years with 24 hour operations.

The second silicon crystal has the shape of a simple parallel piped and was presumed to have non distorted Si (111) diffracting planes. With no thermal distortion, this optic was assumed to contribute nothing to the slope error tolerance.

The last optic was a silicon cylinder (Seso) bent into a toroid by a mechanical bender. Slope errors on these unbent optics were $0.7-0.9$ ? rad rms and with a roughness of $0.46-$ 
$0.58 \mathrm{~nm}$ rms. These values were within specifications. Both the M1 and M2 mirrors were bent with cantilever style spring bending mechanisms (Howells et al., 2000).

\subsection{End Station}

Protein crystallographers have perfected data collection to such a degree that the instrument arrangement around the sample can be defined completely, and the requirement for a large hutch that allows experimental flexibility can be eliminated. The end stations therefore used a mini-hutch configuration with access limited to sample changing through a 90 x $90 \mathrm{~cm}$ sliding door. The mini hutch concept allows instant switch on of the x-ray beam once the sliding door is closed as the hutch is small enough not to require the usual search procedures. Having instant switching on of the beam into the hutch has proved to be a nice feature for users and is similar to the operation of laboratory based x-ray hutches. The sample is mounted on a motorized XYZ stage, which in turn is mounted on a single axis air bearing rotary drive. The air bearing axis rotates much faster than one second per revolution and allows for rapid sample alignment. Being able to rotate the sample 180 degrees in $<0.5 \mathrm{sec}$ allows the collection of true Friedel mates. No overhead is incurred when collecting data in this true "inverse beam" mode, because the detector readout takes longer. As a result, anomalous differences can be calculated from reflections measured in adjacent frames without sacrificing data collection speed. This capability optimizes MAD analysis due to the accuracy of the anomalous differences.

The diffracted x-rays are detected by large area X-ray CCD cameras with active areas of $210 \times 210 \mathrm{~mm}$ or $315 \times 315 \mathrm{~mm}$ (Area Detector Systems Corporation (ADSC)). Fig.6 shows the superstructure of the mini hutch that supports the $315 \times 315 \mathrm{~mm}$ area detector. Around this structure is constructed the separate steel mini hutch radiation enclosure with a footprint of $3 \mathrm{x}$ $1.5 \mathrm{~m}$. The detector is mounted off the gantry structure by means of motorized slides such that the detector face can be positioned between $7-100 \mathrm{~cm}$ from the sample with a 2-theta range of -5 to 45 degrees. The right side of Fig. 6 shows the outline of the sample support, crystal viewing system, and beam monitoring/shutter structure. This section of the mini hutch is independent of the CCD support structure. A three-dimensional model is shown in more detail in Fig. 7, and a close up view is shown in Fig. 8. A section through last $100 \mathrm{~mm}$ of the x-ray path is shown in Fig. 9.

The monochromatic x-ray beam passes through a $50 ? \mathrm{~m}$ thick beryllium window upon entering the minihutch. The Be window seals a helium-filled box that contains the beamline 
photon shutter, beam position monitors, ion chambers, filters and the fast ccd shutter. The beam then passes out into air through a second 50 ?m thick beryllium window (Fig. 9), through a hole in a large $(15 \mathrm{~mm})$ prism, and then through a rapidly exchangeable collimator consisting of a tantalum sheet with pinhole. Collimators with limiting apertures of 100,50 or $30 ? \mathrm{~m}$ are used routinely. The limiting aperture in the collimator is followed $1 \mathrm{~mm}$ beyond by a $300 ? \mathrm{~m}$ tantalum pinhole that acts as a scatter guard. The collimators are pre-aligned and slot in and out for rapid exchange. This arrangement minimizes the time required for users to increase signal to noise by matching the beam size to the crystal size.

The beam passes through a hole in the middle of a small $(5 \mathrm{~mm})$ prism located just in front of the sample. The undiffracted x-rays passing through the sample are trapped by a 0.3 $\mathrm{mm}$ diameter gold back stop supported by a strip of expanded polystyrene packing foam. The diffracted x-rays are barely attenuated by this foam. The foam support is undetectable in the diffraction image; and it shows none of the usual shadows associated with back stop support structures. This foam is in turn supported by a small XY stage that allows for back stop positioning. The arrangement of the 2 prisms (Fig. 9) allows for sample viewing on axis. This feature is particularly critical for alignment of very small crystals. The small prism just before the sample redirects visible light from the sample to a $10 \mathrm{X}$ long- working-distance microscope objective (Mitutoyo), which has a field of view on the TV camera of 580 x 460 ? m. Back illumination is important to see sample contrast, and for this microscope, a fiberoptic light is aimed at the strip of white polystyrene foam that supports the backstop. This material is an excellent scattering source, and it effectively back illuminates the sample with the low coherence required by the imaging microscope. The positioning of the delicate items around the sample (such as the small prism, high magnification microscope, the backstop and the collimator pinhole) are critical to the diffraction measurement. All four of these delicate items are mounted on a substantial XY slide (Fig. 7) that lowers them $200 \mathrm{~mm}$ out of the way prior to sample mounting. This feature allows the goniometer spindle to be completely accessible and sample changes are easily done without disturbing the delicate components of the optical system, the back stop or the collimator. With the stage lowered, the large prism captures the sample image on axis, and the image is relayed to a long-working-distance microscope with a field of view on the TV camera of $4.36 \times 3.28 \mathrm{~mm}$. This view allows for coarse alignment of the sample after initial mounting. When the pinhole/microscope/backstop stage assembly is raised, the sample is viewed with the higher magnification system that enables more precise final alignment. 
There are two pneumatic actuators that can insert devices into the beam in the sample region (Fig. 8). One actuator supports a silicon photodiode that enables monitoring and tune up of the beam. The other supports a rear illuminator light consisting of a fiber optic, a polarization filter and a prism for redirecting the light at the sample. By placing a polarization filter on top of the Mitutoyo high-magnification lens, the sample can be viewed in crossed polarized light in order to help find the crystal and improve alignment accuracy.

\subsection{Beam Position Feedback Scheme}

The focused spot size at the sample is comparable with the 100 ? m defining pinhole. This small beam size presents special problems, because the beam must remain fixed on the sample for long periods of time (hours). Over this time period, we observed slow thermal variations of various beamline components, beam drifts and various other unspecified environmental drifts that caused fluctuations in intensity. At facilities with a larger beam, the problem of drift can be less severe, because beam movements can cause little change in intensity through the limiting pinhole in the endstation. To solve the drift problems, we initially adopted a feedback system based on the horizontal and vertical positional information from split ion chambers located just downstream of the beryllium window at the mini hutch wall. The split ion chambers were located $63 \mathrm{~cm}$ before the focus at the sample. These split ion chambers, however, proved to have a non linear response with both photon energy and beam width, leading them to give an erroneous signal on which to provide beam position feedback. The source of this non linear response is still under investigation.

An alternative feedback scheme was developed. We glued a cerium-doped yttrium aluminum garnet crystal (YAG scintillator) on the shutter blade of the fast CCD shutter (NM Laser) located $17 \mathrm{~cm}$ before the sample. The shutter blade is inclined at 9 degrees to the $\mathrm{x}$-ray beam, and operates by flipping the blade in the vertical. The scintillator image of the beam was viewed with a TV camera which fed the image to a frame grabber that determines the beam position parameters at $15 \mathrm{~Hz}$. Due to the 9 degree angle of the shutter blade and YAG screen with respect to the beam, the scintillator image provides a particularly sensitive and reliable signal for vertical beam position feedback. The device is less sensitive in the horizontal plane, but as the beam is larger in this direction the system is less sensitive to small horizontal beam motions. Vertical beam position was effected by movement of the M2 tilt, and horizontal beam position effected by rolling the second monochromator crystal. Beam position centroid stabilities of $+-5 ? \mathrm{~m}$ are typically achieved. The drawback of this control 
system is that it is only operational when the CCD shutter is closed. When the shutter is open, the system operates in open-loop mode. However, the CCD shutter is generally opened and closed many times per minute during standard data collection runs for protein crystallography. Thus, the beam position is constantly optimized between each diffraction exposure. Such a feedback scheme would not work very well for an XAFS type beamline or other beamlines where the shutter is required to remain open for long (several hours) periods of time.

\section{Beamline Performance}

The calculated and measured fluxes arriving at the sample for all three end stations without a pinhole collimator is shown in Fig. 10. The calculated flux for the inboard and outboard sources is essentially the same up to $\sim 8 \mathrm{KeV}$ and diverges slightly to higher energies. At 12 $\mathrm{KeV}$ the in board (5.29 Tesla source) has $10 \%$ more flux than the outboard (4.37 Tesla) source. The absolute measured flux was determined by cross calibrating a silicon photodiode detector signal with that from a $(\mathrm{NaI}(\mathrm{Tl}))$ scintillator/photomultiplier-tube pulse counting detector (Bicron model 1XMP.040B) operating at $<10^{5} \mathrm{~Hz}$. The low rate of the scintillator detector allows for the assumption of zero dead time and thus linear response. The scintillator detection efficiency is considered to be 1.0 (Holl et al., 1988). All the measured fluxes from the three beamlines are lower than the calculated flux as might be expected as the optics are not perfect. At $12 \mathrm{KeV}$ the flux ratio of measured to calculated for the 3 beamlines is 0.73 , 0.62 and 0.41 for beamlines 8.2.2, 8.2.1 and 8.3.1 respectively. Beamline 8.2.2 has the best performance with a profile similar to the calculated value but slightly lower overall. Beamline 8.2.1 has poorer performance at low energy, whereas 8.3.1 produces less flux at higher energies. The M1 mirror is the only major difference between the three beamlines. Beamline 8.3.1 has the invar M1 mirror, whereas the other two beamlines have silicon M1 mirrors. The metrology measurements indicated that the invar mirror is slightly rougher $(0.9 \mathrm{~nm})$ than the silicon mirrors $(0.5 \mathrm{~nm})$. This slight difference does not readily explain this flux reduction to high energy, because the slight roughness increase would reduce the Strehl ratio but the total flux arriving at the large area silicon photodiode detector (active area $=10 \times 10 \mathrm{~mm}$ ) would be expected to be unaffected. The exact nature of the measured flux loss is still unclear and continues to be investigated.

The focused spot sizes at $12 \mathrm{KeV}$ were measured and found to be $150 \times 100 ? \mathrm{~m}$ FWHM (h $\mathrm{x} \mathrm{v}$ ) for 8.2.1 and 8.2.2 beamlines. These values are consistent with slope errors on the M1, crystal 1 and M2 of 0.8 ? rad rms, as discussed in section 3.1 above. For 8.3.1 the 
focused spot size was measured at 130 x 140 ? m FWHM (h x v), larger in the vertical and smaller in the horizontal when compared to the values calculated by ray tracing. The slightly worse figure specification of the invar M1 mirror (relative to the silicon M1 mirrors on 8.2.1 and 8.2.2) will contribute to the increased vertical spot size, although the magnitude of the figure error does not account for the size increase entirely. In this optical design, changing the M1 or M2 angle allows different aberrations to act against each other. The current optical scheme is designed to eliminate astigmatic coma, and so minimizes the vertical beam size. A small alteration of the M1 or M2 angle breaks this condition, resulting in growth of the vertical beam size. In some alignment conditions, a reduction of the horizontal beam size also is possible. This combination of effects may explain the dimensions of the beam on beamline 8.3.1.

Protein crystallography experiments require a small beam, which is produced by the insertion of a 100 ? m diameter pinhole just before the focus and sample. The measured and calculated flux through the $100 ? \mathrm{~m}$ pinhole is shown in Fig. 11. In general the flux is less than the case with no pinhole as the focused spot size is larger then the pinhole size. Ray tracing indicates that $37 \%$ of the photons should pass through a 100 ?m pinhole. Fig. 12 shows the measured percentage of the flux that passes through the $100 ? \mathrm{~m}$ pinhole for the energy range of the beamline. For all three beamlines, there is a general flux reduction at lower energies. This effect is consistent with beam blow-up in the vertical due to thermal deformation of the first crystal and increased optical aberrations as the vertical fan grows in extent. This explanation is consistent with the water channel distribution in the first monochromator crystal, which was optimized for the $12 \mathrm{KeV}$ diffraction angle. (Section 3.1). Near $12 \mathrm{KeV}$, the flux passing through the pinhole is close to the calculated value (37\%). For beamline 8.3.1. the flux drops for high energy x-rays, consistent with beam blow up in the vertical due to scattering and the reduction in the Strehl ratio due to the slightly worse roughness of the invar mirror used on this beamline.

It should be noted that the beamlines fully satisfy one of the important original goals to provide as good a performance as the beamline 5.0.2 37 pole 2 Tesla wiggler MAD beamline, the 1 st beamline built for protein crystallography at the ALS. The flux through a 100 micron aperture in a 3 mrad convergence angle, at the new beamlines, is around a factor of 3 higher than at the wiggler beamline, even though the total power radiated into the beamline aperture is around a factor of 50 lower.

\section{Control System}


The success of PX beamlines is becoming more dependent on their ease of use, which is strongly influenced by the control software. These beamlines employ separate control systems for the beamline and end station. The beamline is operated by a control system based on Labview (National Instruments), which is becoming standard for beamline control around the ALS. All hardware and software components are modular, because most beamlines contain a limited set of relatively standard components that are independent of the experimental end station. This beamline control system can operate stand-alone and be used to commission the beamline without a functioning end station. Following commissioning, the beamline control system can be put into a driven mode and receive control inputs via the local network from the end station control system. Such commands can shift the energy, control the beam convergence onto the sample and tune up the beamline. The end station control code was developed along two directions. An in-house, end-station control code was developed and deployed on beamlines 8.2.1 and 8.2.2. The 'Blu-Ice' code, available from the Stanford Synchrotron Radiation laboratory (http://smb.slac.stanford.edu/blu-ice/) was deployed on 8.3.1. Both systems support routine data collection. For example, $43 \%$ of the beam time available in November 2002 was used to collect data on beamline 8.3.1. This percentage represents the total time that data was recorded (including the 3 sec. cycle time for downloading the diffraction image from the $\mathrm{CCD}$ ) relative to the total time that photons are coming down the beamline. The remaining $57 \%$ of the time was spent changing samples and choosing experimental strategies.

\section{Future Developments}

Automatic sample change mechanisms that allow for automatic loading and alignment are currently being developed to improve throughput. Sample sizes have continued to shrink, and users routinely use 30 and 50 ?m pinholes. Small crystals also drive the need to increase the flux density at the sample. To this end, planned improvements to the ALS ring, such as top off mode injection, will provide $2-3$ times the present average current with a smaller vertical beam size. To benefit from this improved source brightness, the possible options include replacing the M1 mirrors with internally cooled silicon mirrors, cryocooling the first monochromator crystal, and replacing the M2 mirrors with a conical design to provide improved vertical focusing.

\section{Conclusions}


Three warm bend magnets at the ALS were replaced with superconducting 6 Tesla bend magnets that produce synchrotron radiation with a critical energy of $12 \mathrm{KeV}$ from the lowenergy, $1.9 \mathrm{GeV}$ ALS ring. A suite of three protein crystallography beamlines was installed using a single superbend source. The low power of the source allows for relatively inexpensive beamline construction costs. The high brightness of the source is well matched to the requirements of protein crystallography, such that the available photons are used efficiently. The beamlines are currently operating with flux rates at the sample of $2-3 \times 10^{11}$ photons/sec/400mA with $3 \mathrm{mrad}$ horizontal divergence through a $100 ? \mathrm{~m}$ pinhole. This flux is approximately three times higher than that of the ALS 5.0.2 wiggler beamline.

\section{Acknowledgements}

The Advanced Light Source is supported by the Director, Office of Science, Office of Basic Energy Sciences, Materials Sciences Division, of the U.S. Department of Energy under Contract No. DE-AC03-76SF00098 at Lawrence Berkeley National Laboratory. The Howard Hughes Medical Institute provided funding for beamlines 8.2.1 and 8.2.2. Beamline 8.3.1 was funded by the National Science Foundation, the University of California, Berkeley, the University of California, San Francisco and Henry Wheeler.

\section{Appendix}

The focal lengths, $f$, of a toroidal mirror in the tangential and sagittal directions (indicated by subscripts $s$ and $t$ ) are given by Coddington's equations

$$
\frac{1}{r_{t}} ? \frac{1}{r_{t} ?} ? \frac{1}{f_{t}} ? \frac{2}{R \cos ?} \text { and } \frac{1}{r_{s}} ? \frac{1}{r_{s} ?} ? \frac{1}{f_{s}} ? \frac{2 \cos ?}{?}
$$

where $r$ and $r$ : are the object and image distances respectively, $R$ is the major radius, ? the minor radius and ? is the angle of incidence measured from the normal. It is possible to choose $R$ and ? so that the tangential and sagittal foci coincide in which case the image is said to be stigmatic. It has long been known that, for the special case of a toroidal mirror imaging a point source to a nominal point image, at unity magnification $\left(r_{s} ? r_{t} ? r_{s}^{? ? ?} r_{t}\right)$, the image quality is exceptionally high. This is due to the fact that the comatic aberrations (sagittal and tangential) vanish under this condition. Now, based on a systematic ray tracing study (Padmore, 2000), we have learned that a grazing-incidence toroidal mirror gives unusually good image quality for another, hitherto unsuspected, "magic" set of object and image conjugates. In the new magic configuration, the object-side beam is on-axis but astigmatic, which means in general that the source consists of two mutually perpendicular lines at 
different distances $r_{s}$ and $r_{t}$ from the mirror. The beneficial effect occurs under the following particular conditions

$r_{s}$ ? arbitrary, $r_{t} ? ?, r_{s}^{? ? ?} r_{s} / 2, r_{t}^{?} ? ? r_{s}^{?}$

in which one of the source lines is at infinity and the other lies in the symmetry plane of the toroidal mirror. These are just the conditions that are commonly used on x-ray beamlines, such as the one reported in this paper, where the sequence (1) vertically-collimating cylinder mirror, (2) double-crystal monochromator, (3) toroidal focusing mirror, is used to deliver a stigmatic image to the experiment. For such beamlines, the toroidal-mirror has $r_{t}$ equal to infinity due to collimation and $r_{s}$ equal to the collimator-mirror-to-synchrotron-source distance. We have studied this imaging scheme using an optical-path-function analysis and have shown that for such a source configuration, the normal point-to-point path-function analysis (Padmore, et al., 1998) is insufficient and an extension of the theory is required to describe the line source implied in (A2). We have provided an appropriate extension of the theory and used it to show that the coma terms of the optical-path-function expansion under the magic conditions are as follows

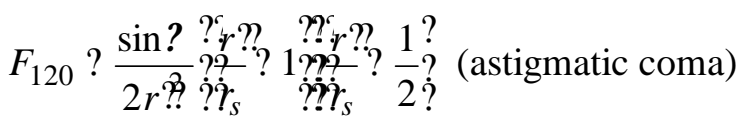

$F_{300} ? \frac{\cos ^{2} ? \sin ?}{2 r ? !}$ (primary coma)

where ? is the incidence angle to the normal and we have dropped the $s$ and $t$ subscripts for $r$ ? since the imaging is stigmatic on the image side. As can be seen the principal aberration, the astigmatic coma, indeed vanishes at $r ? ? r_{s} / 2$ while the primary coma, although not exactly zero, can be sufficiently small. More specifically, the marginal-ray aberration due to primary coma, which is a very conservative measure, equals $41 \mu \mathrm{m}$ for the case of interest here. Thus, in summary, the good optical performance of this beamline is predicted by ray trace, explained by aberration analysis and delivered by the constructed beamline as shown by the xray measurements reported in the main text.

\section{References}

1-2 GeV Synchrotron Radiation Source - Conceptual Design Report, Lawrence Berkeley National Laboratory Report PUB-5172. July 1986.

ADSC, 12550 Stowe Drive, Poway, California 92064, USA

Acteron Corp., 1000 Commercial Street, San Carlos CA 94070, USA. 
Bicron model 1XMP.040B scintillator detector - from Saint-Gobain Crystals \& Detectors, 12345 Kinsman Road, Newbury, OH 44065, USA.

Earnest, T., Padmore, H.A., Cork, C., Behrsing, R. \&Kim, S-H. (1996). Journal of

CrystalGrowth. 168, 248-252.

Épotecny, 9 Street Aristide Briand, 92300 Levallois-Perret, France.

Freund, A. (2000). Private Communication.

Garman E. Nave C. (2002). Journal of Synchrotron Radiation. 9, 327-328.

Glaeser, R., Facciotti, M., Walian, P., Rouhani, S., Holton, J., MacDowell, A., Celestre, R., Cambie, D. \& Padmore, H. (2000). Biophysical Journal, 78, 3178-3185.

Henderson, R. (1990). Proc.R.Soc.Lond.Biol. 241, 6-8.

Holl, I., Lorenz, E. and Mageras, G. (1988) IEEE Tans. Nuclear Science, 35 105-109. This paper indicates that the visible photon yield from $\mathrm{NaI}(\mathrm{Tl})$ scintillator is 38 photons $/ \mathrm{KeV}$ when irradiated with x-rays. We assume a conservative light coupling of these photons into the photomultiplier tube of $\sim 50 \%$. The efficiency of the Hamamatsu R580 photomultiplier tube is $\sim 25 \%$ (http://usa.hamamatsu.com) at the emission wavelength of $\mathrm{NaI}(\mathrm{Tl})-430 \mathrm{~nm}$. A $12 \mathrm{KeV}$ $\mathrm{x}$-rays is expected to generate $\sim 57$ electrons/x-ray from the photocathode before entering the electron multiplier section. The scintillator photomultiplier combination is thus expected to single count $\mathrm{x}$-rays with a quantum efficiency of 1.0 after the absorption by the $125 \mu \mathrm{m}$ thick beryllium entrance window is taken into account.

Howells, M.R. (1999). Advanced Light Source Notes. LSBL-529.

Howells, M.R., Cambie, D., Duarte, R., Irick, S., MacDowell, A.A., Padmore, H.A., Renner, T., Seungyu, R. \& Sandler, R. (2000). Optical Engineering. 39, 2748-2762.

Howells, M.R., Burt, P., Cambie, D., Duarte, R., Franck, A., Irick, S., MacDowell, A.A., MacGill, D., Paquin, R. \& Plate, D. (2002). SPIE Proceedings 4782 94-103.

Howells, M.R., MacDowell, A.A., McKinney, W.R. \& Padmore, H.A. (2003). To be submitted to J.Synch. Rad.

InSync, 2511C Broadbent Parkway NE, Albuquerque, NM 87107, USA.

Kohzu Seiki Co., 2-27-37 Mishuku, Setagaya-Ku, Tokyo, 154-0005, Japan.

Lai, B. \& Cerrina, F. (1986). Nucl. Instrum. Meth. A246, 337-341.

Mitutoyo Corp., 965 Corporate Boulevard, Aurora, IL 60504, USA.

National Instruments, 11500 N.Mpoac Expwy, Austin , Tx 78759, USA.

NM Laser Products Inc., 140 San Lazaro Ave. Sunnyvale, CA 94086, USA.

Padmore, H.A. (2000). Advanced Light Source Notes. LSBL-542. 
Padmore, H.A., Howells, M.R. \& McKinney, W.R., Vacuum Ultraviolet Spectroscopy II, Ed. Samson, J.A.R., Academic Press, (1998) Chap.2, 21-54.

Robin, D., Biocca, A., Brown, B., Byrne, W., Calais, D., Chin, M., Colomb, D., Corradi, C., De Vries, J., Fahmie, M., Geyer, A., Harkins, J., Jacobson, S., Krupnick, J., Marks, S., McDonald, J., Mueller, R., Ottens, F., Paterson, A., Pipersky, P., Ritchie, A., Schlueter, R., Spring, J., Steier, C., Taylor, C., Thur, W., Timossi, C., Wandesforde, A., Zbasnik, J., Chen \& J., Wang, B. (2001). Proc. of the Particle Accelerator Conference, Chicago, IEEE, 26322634.

Robin, D., Benjegerdes, R., Biocca, A., Bish, P., Brown, W., Calais, D., Chin, M., Corradi, C., Coulomb, D., De Vries, J., DeMarco, R., Fahmie, M., Geyer, A., Harkins, J., Henderson, T., Hinkson, J., Hoyer, E., Hull, D., Jacobson, S., Krupnick, J., Marks, S., McDonald, J., Mollinari, P., Mueller, R., Nadolski, L., Nishimura, K., Ottens, F., Paterson, J.A., Pipersky, P., Ritchie, A., Rossi, S., Salvant, B., Schlueter, R., Schwartz, A., Spring, J., Steier, C., Taylor, C., Thur, W., Timossi, C., Wandesforde, J. \& Zbasnik, J..(2002). J. Proceedings of the 2002 European Particle Accelerator Conf., Paris, France, 215-217.

Tamura, L. \& Robinson, A. (2002). Synchrotron Radiation News, 15, 30-34.

Takacs, P.Z \& Qian, S. (1989). United States Patent 4884697.

Seso, 305, rue Louis Armand - B.P. 55000, 1372 Aix-en-Provence, Cedex 3, France

Steier.C., Robin, D.S., Wu, Y., Decking, W., Laskar, J., \& Nadolski, L., (2001). Proc. of the Particle Accelerator Conference, Chicago, IEEE, 434-436

Yusupov, M.M., Yusupova, G.Z., Baucom, A., Lieberman, K., Earnest,T.N., Cate,J.H.D. \& Noller, H.F. (2001). Science. 292,883-896. 


\section{Figures}
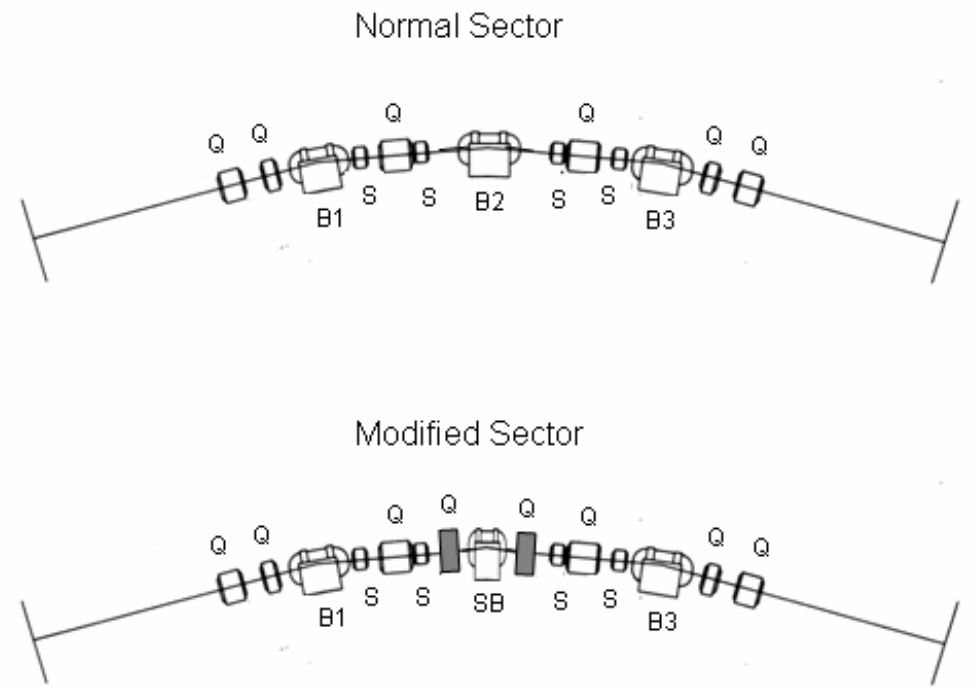

Figure 1. Magnet layout of the regular warm (top) and modified superbend (bottom) sector. Key:- BN=Bending Magnet \#N, SB=Superbend, Q=Quadrupole, S=Sextupole. 

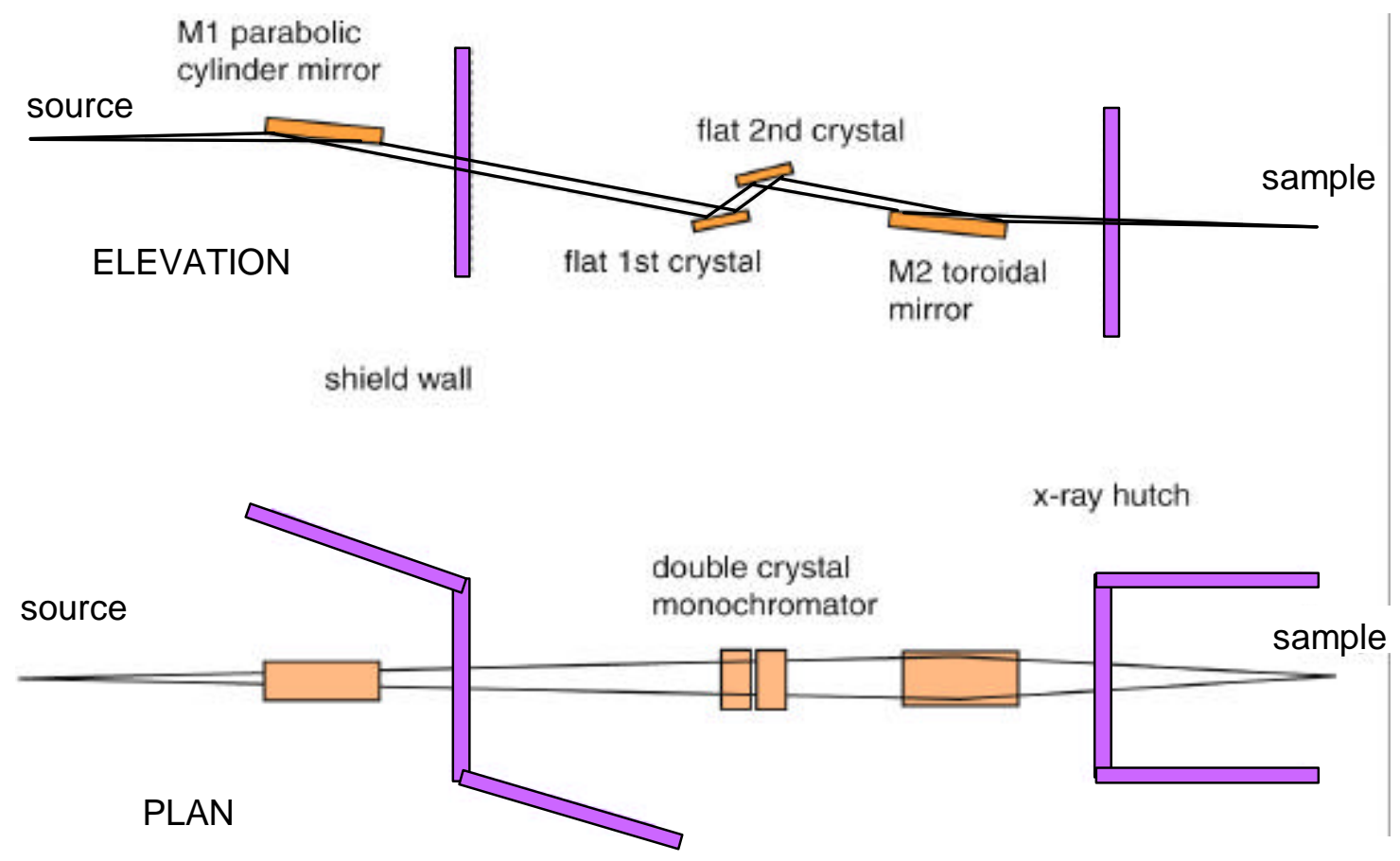

Figure 2. Schematic layout of the new protein crystallography beamlines with a superbend dipole magnet source. The beamline acceptance is $1.5 \times 0.5 \mathrm{mrad}(\mathrm{h} \mathrm{x} \mathrm{vl})$. The plane parabola mirror is $6.5 \mathrm{~m}$ from the source. For the three beamlines, the samples are $26,29.3$ and $32.3 \mathrm{~m}$ from the source. The toroidal M2 mirror demagnifies in the horizontal in a 2:1 ratio. The monochromators are typically $16 \mathrm{~m}$ from the source. 


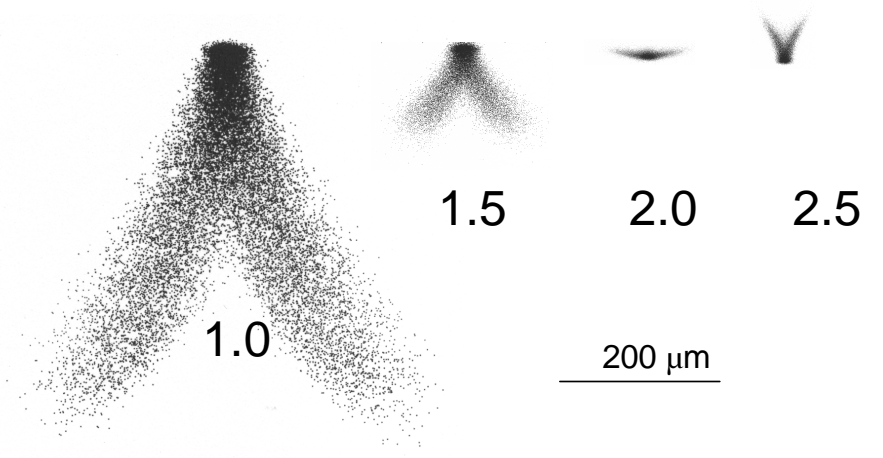

Figure 3. Spot ray traces of the beamline optical layout of Fig. 2 for the indicated horizontal demagnifications . Source size $=230 \times 23 ? \mathrm{~m}$ FWHM. Vertical acceptance $=0.5 \mathrm{mrad}$.

Horizontal acceptance $=(3.0 /$ demagnification $)$ mrad. Vertical demagnification $=0.41,0.51$, 0.62 and 0.72 for demagnifications $1.0,1.5,2.0$ and 2.5 respectively. The demagnification of 2.0 compensates for aberrations introduced by the individual optical elements. 


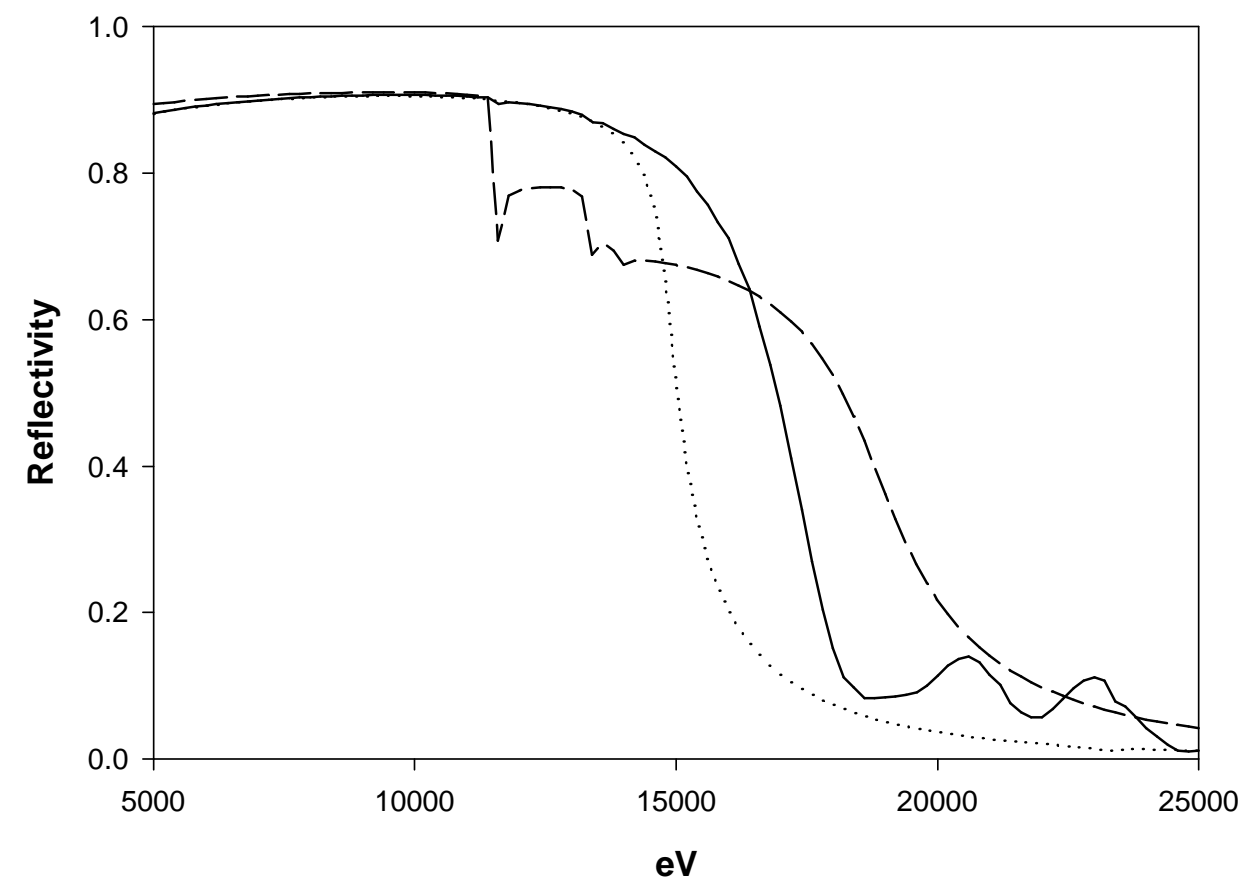

Figure 4. Calculated reflectivity plots of a bilayer consisting of 8 nm Rhodium over $25 \mathrm{~nm}$ platinum (solid) and for single layers of platinum (dashed) and rhodium (dot) at $4.5 \mathrm{mrad}$ grazing angle. The bilayer extends the energy range cut off beyond that for a simple rhodium mirror whilst reducing the effects of the platinum $\mathrm{L}$ absorption edges. 

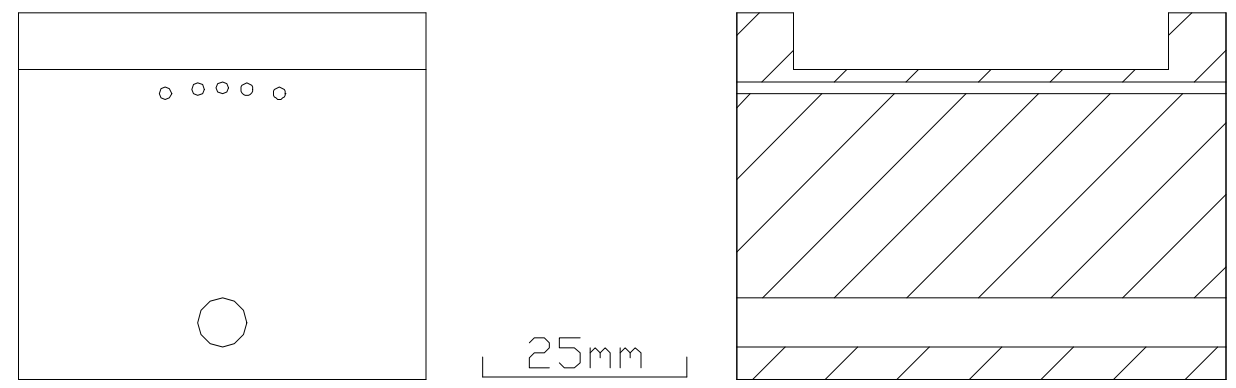

Figure 5. Sections through the first $\operatorname{Si}(111)$ crystals used in the monochromator. The holes are $1.5 \mathrm{~mm}$ diameter through the $60 \mathrm{~mm}$ thick crystal. The minimum hot wall thickness is 1.5 $\mathrm{mm}$, the holes are separated 3 and $4 \mathrm{~mm}$ apart and located on the arc of a $38 \mathrm{~mm}$ radius circle. 


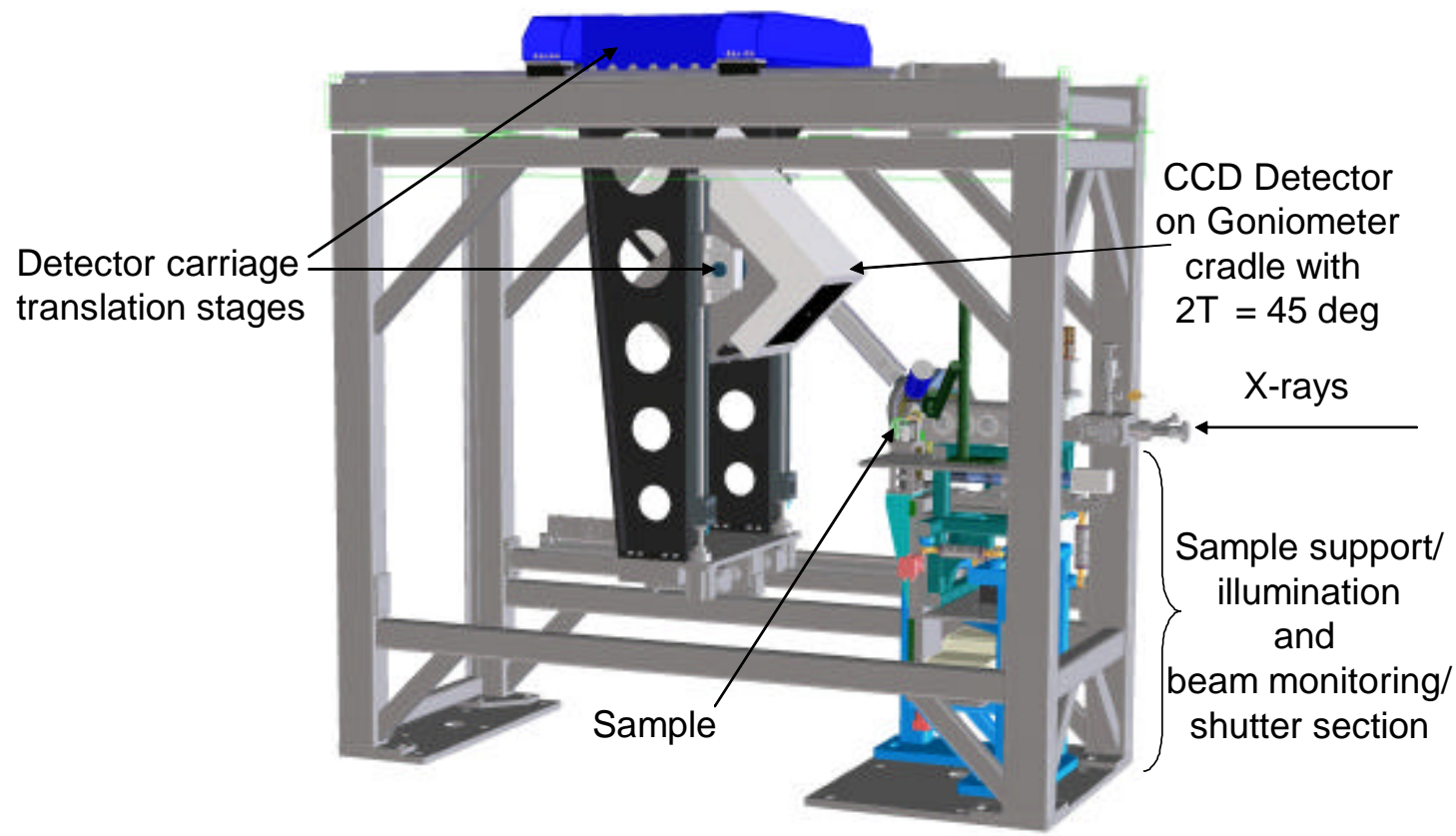

Figure 6. Superstructure of the gantry system that supports the CCD detector. The detector face can be positioned $7-100 \mathrm{~cm}$ from the sample, and the 2 -theta angle can be varied between -5 to $45 \mathrm{deg}$. The three degrees of freedom are accomplished using the two translation devices shown, with a rotation follower pivoted about the sample rotation axis that constrains the detector face to point at the sample. The entire structure is enclosed in a steel box that comprises the mini hutch. 


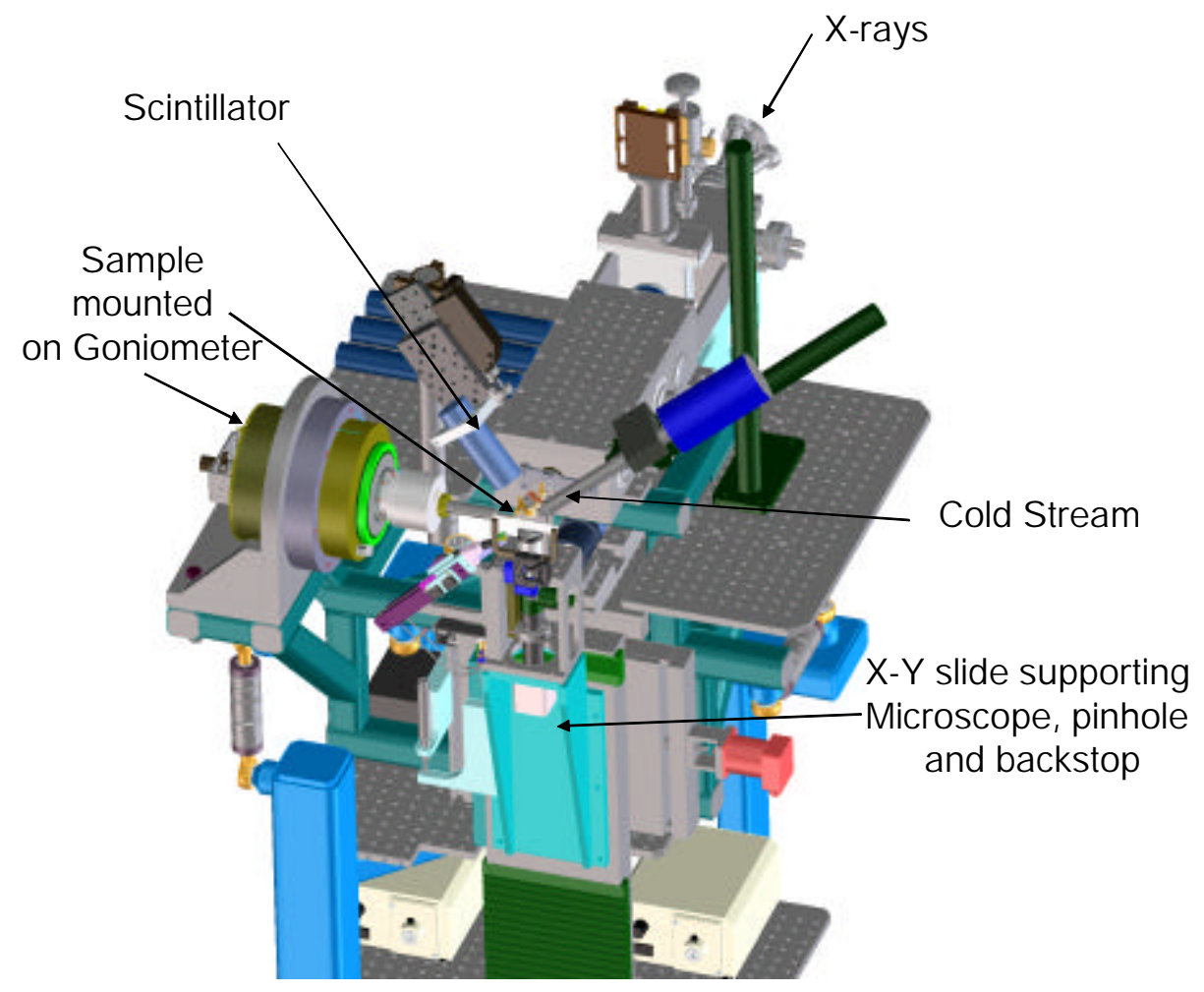

Figure 7. View of the arrangement and support structure for the sample and the various beam conditioning elements. 


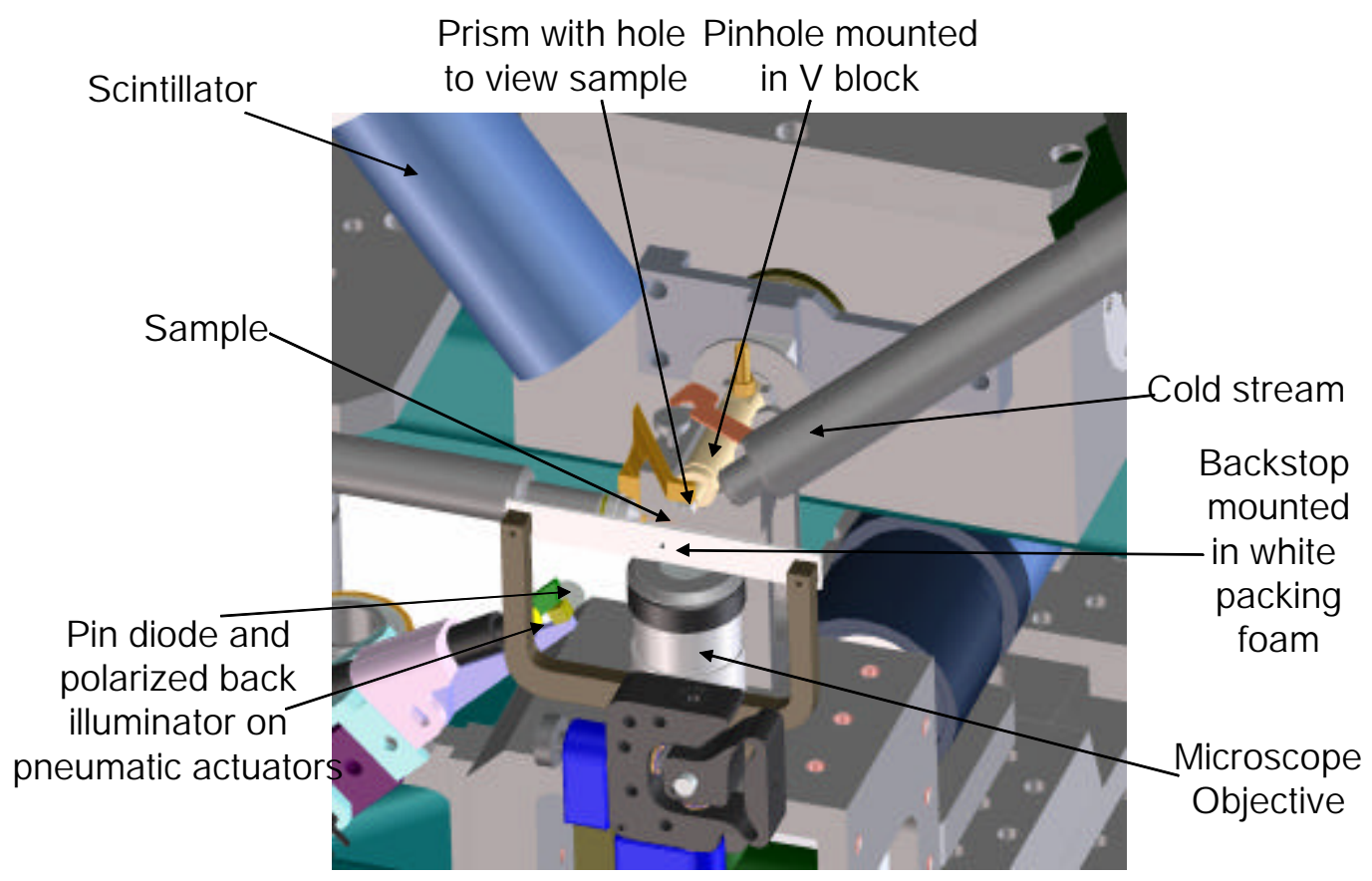

Figure 8. Close up view of the experimental arrangement around the sample. 


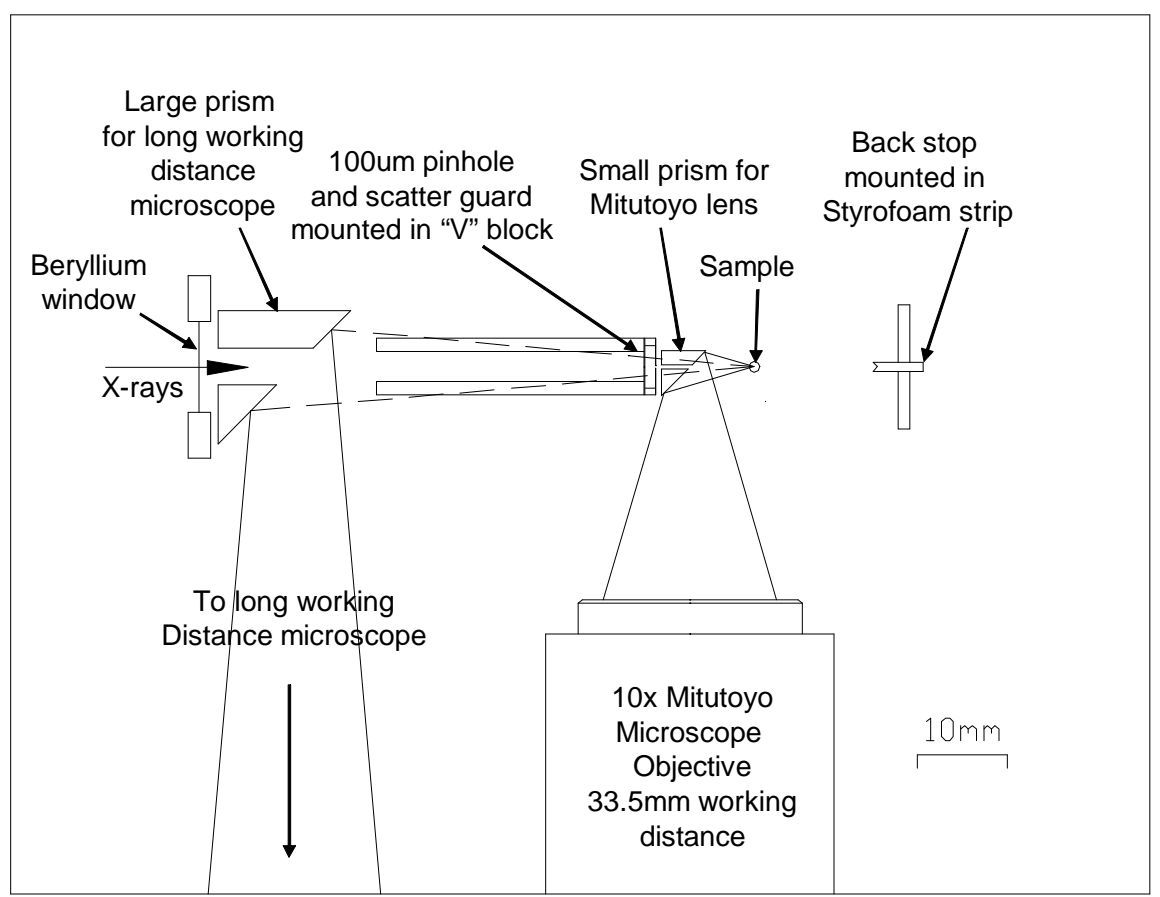

Figure 9. Schematic section through the last 100 mm flight path of the x-rays in air. 


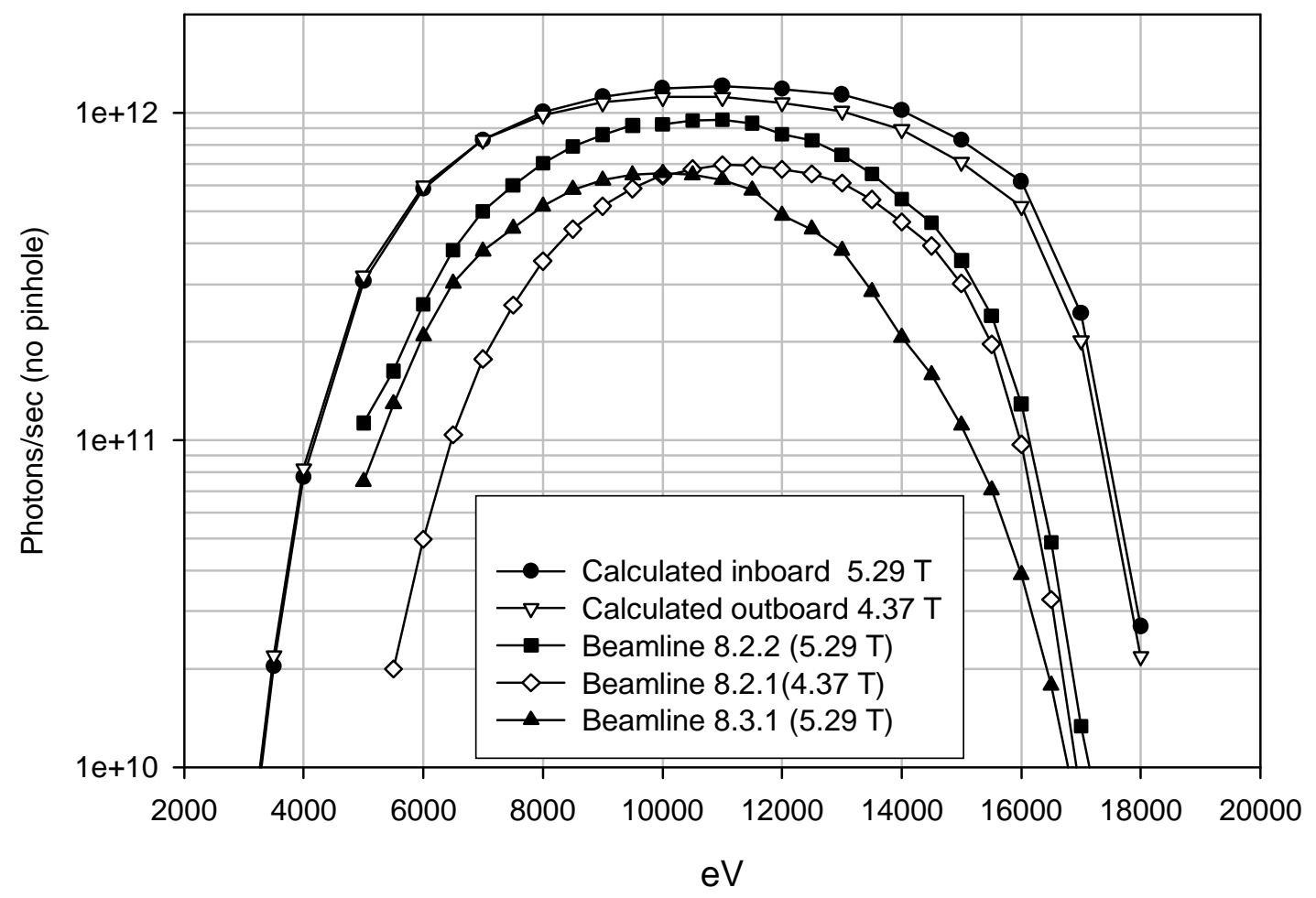

Figure 10. Calculated and measured total flux of the beamlines at the sample position (no pinhole). The calculated flux is for the inboard (4.37 Tesla) and outboard (5.29 Tesla) superbend dipole magnet sources. Flux rates are for $400 \mathrm{~mA}$ in the ALS and full acceptance of the beamline resulting in a convergence onto the sample of $3.0 \mathrm{mrad}(\mathrm{h}) \times 0.31 \mathrm{mrad}(\mathrm{v})$. 


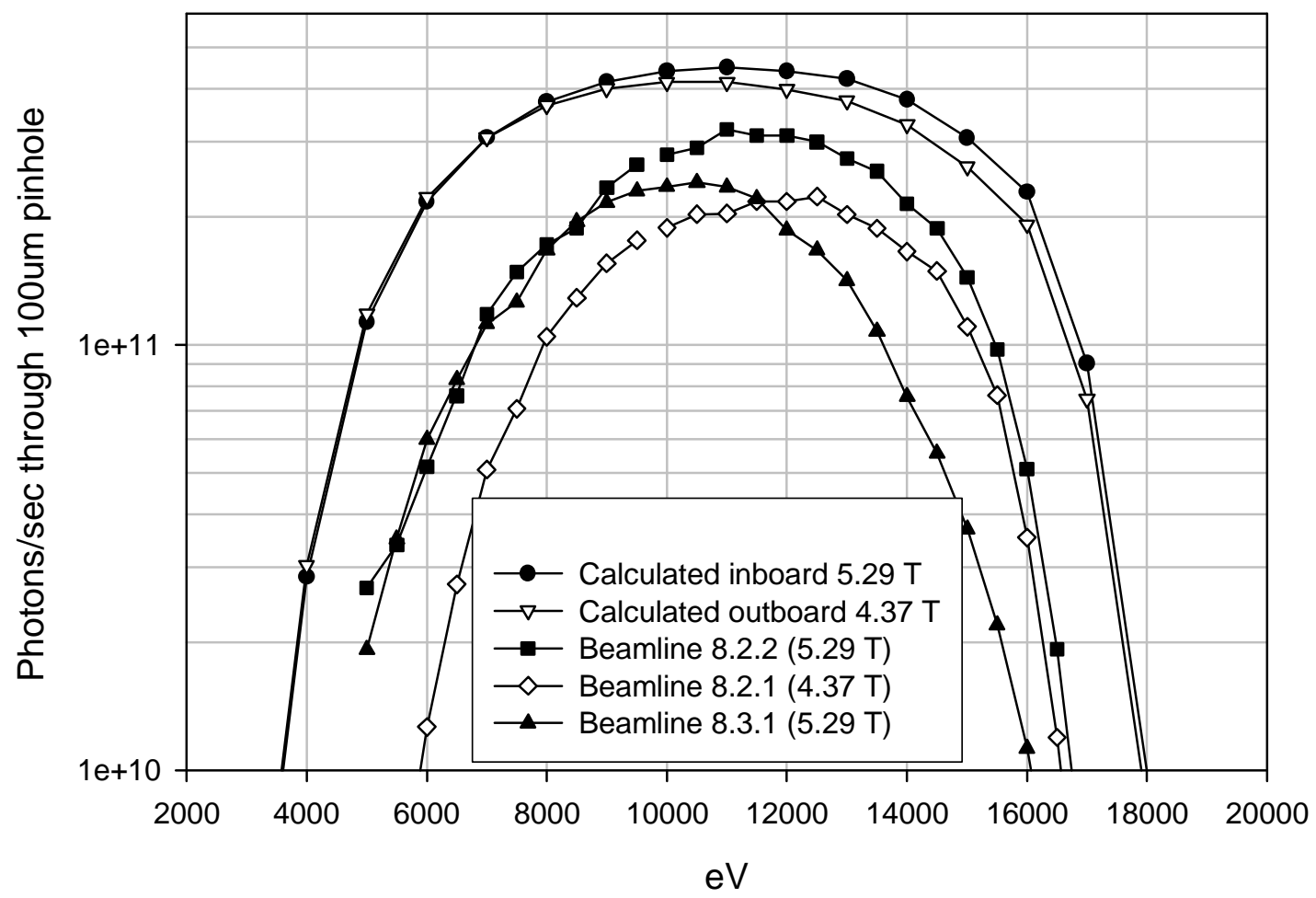

Figure 11. Plot of calculated and measured flux though a $100 ? \mathrm{~m}$ pinhole at the sample position. Flux rates are for $400 \mathrm{~mA}$ in the ALS and full acceptance of the beamline resulting in a convergence onto the sample of $3.0 \mathrm{mrad}(\mathrm{h}) \mathrm{x} 0.31 \mathrm{mrad}(\mathrm{v})$. 


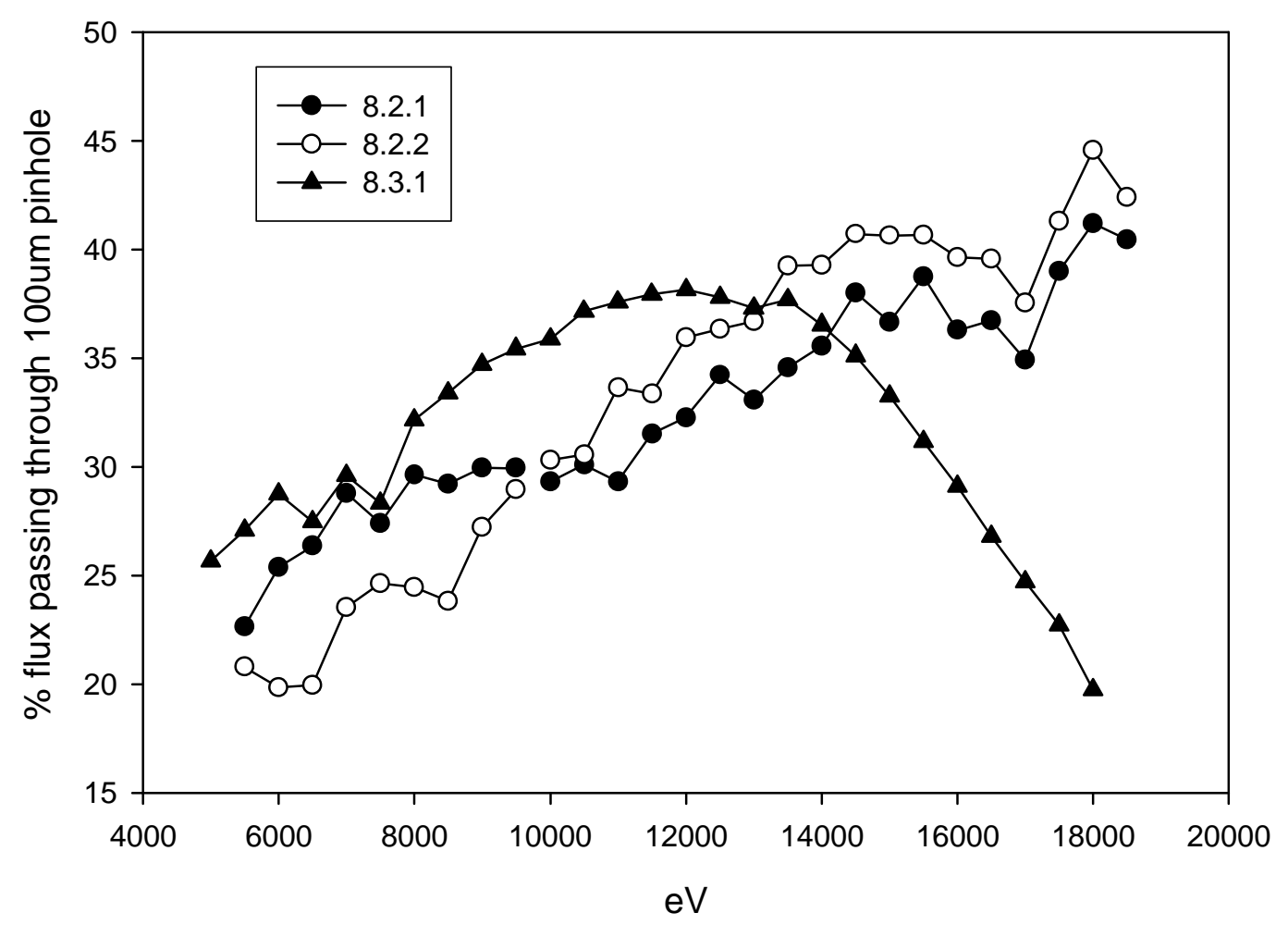

Figure 12. Plot of percentage of $\mathrm{x}$-rays that pass through the $100 ? \mathrm{~m}$ pinhole versus energy. This measurement is for the full horizontal $(1.5 \mathrm{mrad})$ and vertical $(0.5 \mathrm{mrad})$ acceptance of the beamline 